\title{
Les urgences : une solution et non un problème !
}

\section{Emergency: a solution, not a problem!}

\author{
B. Riou \\ (C) SFMU et Springer-Verlag France 2013
}

Les urgences sont devenues une préoccupation importante des politiques, des médias, des administrateurs de la santé et des experts de l'organisation de la santé. Cet intérêt croissant pourrait satisfaire les urgentistes s'il ne prenait une direction parfois inquiétante qui semble à la fois se fourvoyer (mauvais traitement !) et constituer une erreur d'analyse profonde de l'évolution de notre système de soins (mauvais diagnostic !). Aujourd'hui tout le monde semble se focaliser sur le flux de patients qui se présentent aux urgences. Tel député se proposait de résumer l'évaluation des services d'urgences à la mesure du temps de passage des patients. Telle Agence régionale de santé (ARS) se proposait de mettre au cœur de sa stratégie la baisse du recours aux urgences. Tel colloque sous le haut patronage de la ministre des Affaires Sociales et de la Santé mettait à son programme le thème «Urgences : entrée interdite ». Même l'enquête prochaine de la Direction de la recherche des études de l'évaluation et de la statistique (DREES) se proposait d'étudier en priorité les motifs de recours aux urgences. Bref il y a trop de patients aux urgences, ils n'ont rien à y faire, ils attendent trop longtemps. Et cela alors même que l'analyse de la littérature scientifique montre l'inanité de la notion de « patient non urgent » aux urgences [1].

Pourtant, le flux de patients n'est pas le problème majeur des urgences aujourd'hui. Le problème majeur est de trouver des lits d'hospitalisations pour des patients qui ne peuvent plus ou pas être pris en charge en médecine ambulatoire. Le scandale c'est tous ces patients qui passent parfois 24 à 48 heures sur un brancard aux urgences dans des conditions

\section{B. Riou $(\square)$}

Service d'accueil des urgences, CHU Pitié-Salpêtrière, Assistance publique-hôpitaux de Paris, 47-83, boulevard de l'Hôpital, F-75651 Paris cedex 13, France Université Pierre et Marie Curie-Paris 6, F-75005 Paris e-mail : bruno.riou@psl.aphp.fr

\footnotetext{
${ }^{1}$ Texte présenté dans le cadre des $1^{\text {res }}$ Assises de l'urgence, 13 septembre 2012, Paris. L'ensemble des textes est disponible sur http:// www.samu-de-france.fr/documents/actus/129/732/sudf 1-assisesde-1-urgence_txt-et-reco_120913_vf.pdf (dernier accès le 20 décembre 2012)
}

inacceptables et qui constituent sur le plan médical une véritable perte de chance, surtout pour les plus âgés, ce qui n'empêche pas les hôpitaux de facturer cette «prestation » au prix fort. L'énergie que dépensent les équipes des urgences pour trouver des lits, véritable travail de Sisyphe, peu valorisant et épuisant, détourne une partie non négligeable du temps médical et paramédical qui pourrait être consacré plus utilement à la prise en charge. Mais qui se préoccupe de ce problème?

Vouloir réduire le nombre de patients qui se présentent aux urgences peut paraître parfaitement logique avec des arguments imparables comme la nécessité de revaloriser la médecine ambulatoire, de favoriser l'interaction villehôpital, de développer l'hospitalisation à domicile, toutes choses belles et bonnes. On se trompe juste de point d'impact et de stratégie. C'est comme si devant les foules qui se présentent à Pôle emploi (trop nombreuses, qui n'ont rien à y faire ou pour qui on ne peut rien faire, qui attendent trop !), on décidait des mesures pour les empêcher de s'y rendre, oubliant juste les raisons qui produisent de nouveaux chômeurs et celles qui les empêchent de retrouver du travail. Et on imagine le tollé si d'aventure certains proposaient aux chômeurs de consulter d'abord leur chasseur de tête référent ou si on tentait de promouvoir le concept de chômage « ressentie » comme a été promu celui d'urgence ressentie. Vouloir diminuer le nombre de patients qui se présentent aux urgences, c'est supposer qu'il existe une alternative, en fait le plus souvent absente du fait de l'effondrement progressif et inquiétant de la médecine générale libérale et qui ne peut que s'aggraver dans les années qui viennent. C'est supposer que les patients peuvent trouver un accès facile à un spécialiste, sans dépassement d'honoraire, ne serait-ce que pour une simple radiographie. C'est supposer qu'un médecin libéral peut faire hospitaliser facilement un patient qui ne rentre pas dans les cases rentables de la T2A, et ils sont nombreux. C'est supposer qu'un psychiatre a d'autre solution que d'adresser son patient aux urgences en cas de crise. Le flux des patients qui consultent aux urgences est un symptôme des dysfonctionnements de notre système de santé. Il faut s'attaquer à ces dysfonctionnements et non au symptôme. Vouloir créer une maison médicale de garde (MMG) est 
une bonne chose si l'objectif est d'améliorer l'offre de soins de médecine ambulatoire, et son évaluation doit se faire sur ce critère. C'est une erreur si l'objectif est de diminuer le flux aux urgences. D'ailleurs, les MMG n'ont jamais diminué le flux de patients aux urgences. La médecine a évolué et la volonté de réduire le flux de patients aux urgences n'est guère compatible avec les campagnes de sensibilisation du grand public aux douleurs thoraciques pour la prise en charge des infarctus du myocarde ou celle sur les accidents vasculaires cérébraux (faites le 15 !). Il est préférable d'accueillir « inutilement » dans un service d'urgence dix patients ayant une douleur thoracique bénigne pour permettre de mettre le patient ayant un infarctus du myocarde à temps entre les mains du cardiologue interventionnel.

Et si on changeait de paradigme : les urgences sont une solution et non un problème [2]. Il convient d'abord de remarquer que le recours croissant aux urgences n'est pas propre à la France mais se retrouve dans la plupart sinon tous les pays [2]. D'ailleurs le temps d'attente dans les urgences françaises se compare favorablement avec ce qui se passe dans d'autre pays et non des moindres. Notre système de santé, comme celui d'autres pays, a évolué en modifiant profondément la médecine ambulatoire d'une part et le secteur hospitalier d'autre part. L'hôpital s'est concentré et se concentrera encore plus autour de plateaux techniques lourds, s'est converti à l'hôpital de jour et de semaine, s'est rempli jusqu'à dépasser le seuil de remplissage critique audelà duquel il n'y a plus de marges de manœuvre pour les imprévus. Face à cette évolution, les urgences modernes jouent un rôle croissant, véritable centre de tri et d'orientation des patients (le « hub »), dont le fonctionnement est devenu vital pour l'ensemble. Les services médicochirurgicaux n'ont plus les moyens de gérer leurs propres urgences et heureusement que les urgences et leur unité d'hospitalisa- tion de courte durée (UHCD) sont là pour y pallier. Le débat sur la médecine ambulatoire en nuit profonde est probablement déjà clos même si tous les acteurs ne l'ont pas encore compris. Seules les structures d'urgences (Samu-Centre 15 -service des urgences) seront présentes demain pour les prendre en charge. Les Samu-Centre 15 jouent un rôle crucial dans la réponse immédiate qui peut être apportée à un appel et l'orientation des patients et ce rôle ne fera que s'accentuer avec les restructurations hospitalières inévitables en cours, que ce soit pour des raisons de raréfaction des plateaux techniques de plus en plus complexes, des raisons économiques ou de démographie médicale. Il est contreproductif et dangereux que cette activité essentielle soit la cible d'ajustement budgétaire ou de politiques visant à en diminuer l'activité.

Notre système de santé connaît des difficultés importantes et la crise économique ne laisse guère d'espoir sur des augmentations de moyens. Les urgences peuvent et doivent s'améliorer. Encore convient-il de ne pas se tromper de diagnostic ou de traitement et de ne pas réformer les urgences alors que les problèmes sont en amont et en aval. Il convient de considérer dorénavant que les structures d'urgences préhospitalières et hospitalières sont une solution aux difficultés de notre système de santé et non un problème. Encore faut-il rendre ces structures attractives pour le personnel médical et paramédical qui y travaille. Là est l'urgence !

\section{Références}

1. Durand AC, Gentile S, Devictor B, et al (2011) ED patients: How non urgent are they? Systematic review of the emergency literature. Am J Emerg Med 29:333-45

2. Schuur JD, Venkatesh AK (2012) The growing role of emergency departments in hospital admissions. N Engl J Med 367:391-3 